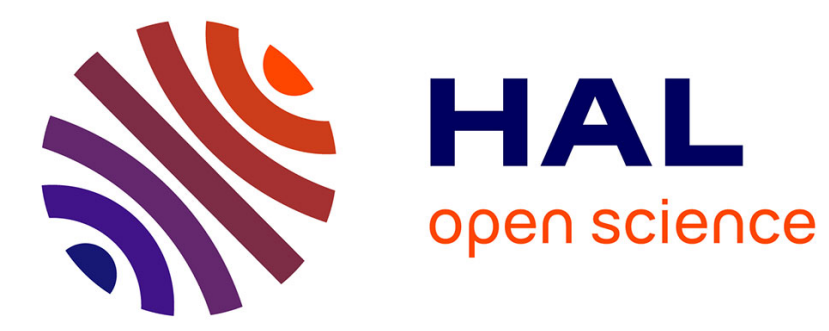

\title{
Numerical Tracking of Limit Points for Direct Parametric Analysis in Nonlinear Rotordynamics
}

Lihan Xie, Sébastien Baguet, Benoit Prabel, Régis Dufour

\section{To cite this version:}

Lihan Xie, Sébastien Baguet, Benoit Prabel, Régis Dufour. Numerical Tracking of Limit Points for Direct Parametric Analysis in Nonlinear Rotordynamics. Journal of Vibration and Acoustics, 2016, 138 (2), pp.021007. 10.1115/1.4032182 . hal-01265919

\section{HAL Id: hal-01265919 \\ https://hal.science/hal-01265919}

Submitted on 1 Feb 2016

HAL is a multi-disciplinary open access archive for the deposit and dissemination of scientific research documents, whether they are published or not. The documents may come from teaching and research institutions in France or abroad, or from public or private research centers.
L'archive ouverte pluridisciplinaire HAL, est destinée au dépôt et à la diffusion de documents scientifiques de niveau recherche, publiés ou non, émanant des établissements d'enseignement et de recherche français ou étrangers, des laboratoires publics ou privés. 


\title{
Numerical Tracking of Limit Points for Direct Parametric Analysis in Nonlinear Rotordynamics
}

\author{
Lihan Xie \\ Université de Lyon, CNRS, INSA-Lyon, LaMCoS, UMR5259 F-69621 France/ \\ CEA-Saclay, DEN, DANS, DM2S, SEMT, DYN F-91191 France \\ Email: lihan.xie@insa-Iyon.fr \\ Sébastien Baguet * \\ Université de Lyon, CNRS, INSA-Lyon, LaMCoS, UMR5259 F-69621 France \\ Email: sebastien.baguet@insa-lyon.fr \\ Benoit Prabel \\ CEA-Saclay, DEN, DANS, DM2S, SEMT, DYN F-91191 France \\ IMSIA, UMR9219, CNRS-EDF-CEA-ENSTA, Université Paris Saclay \\ Email: benoit.prabel@cea.fr \\ Régis Dufour \\ Université de Lyon, CNRS, INSA-Lyon, LaMCoS, UMR5259 F-69621 France \\ Email: regis.dufour@insa-lyon.fr
}

\begin{abstract}
A frequency-domain approach for direct parametric analysis of limit points of nonlinear dynamical systems is presented in this paper. Instead of computing responses curves for several values of a given system parameter, the direct tracking of limit points is performed. The whole numerical procedure is based on the Harmonic Balance Method and can be decomposed in three distinct steps. Firstly, a response curve is calculated by HBM combined with a continuation technique until a limit point is detected. Then this starting limit point is used to initialize the direct tracking of limit points which is based on the combination of a so-called extended system and a continuation technique. With only one computation, a complete branch of limit points is obtained, which provides the stability boundary with respect to system parameters such as nonlinearity or excitation level. Several numerical examples demonstrate the capabilities and the performance of the proposed method.
\end{abstract}

\section{INTRODUCTION}

Generally speaking, the rotating systems utilized in the energy production have a small rotor-stator gap, are able to run during long periods, and are mounted on hydrodynamic bearings. Rotor-stator interactions in case of blade loss, crack propagation due to fatigue, and a variable stiff-

* Address all correspondence for other issues to this author. ness due to the nonlinear restoring forces of the bearings can make the rotordynamics nonlinear and the responses complicated: significant amplitude and frequency shifts are introduced, sub- and super-harmonics appear, and hysteresis occur. It is of great importance to understand, predict and control this complicated dynamics. For low cost, repeatability, and operability reasons, this is commonly achieved by means of numerical simulation. The literature comprises a lot of numerical investigations for various nonlinear properties, such as rotor-stator contact [1] [2], crack breathing [3] [4], hydrodynamic bearings [5] [6], on-board rotor mounted on hydrodynamic bearings [7], etc.

In order to compute solutions to such problems, time integration methods are commonly used. However, for steadystate periodic solutions, specific methods such as the shooting method [8] or the frequency-domain Harmonic Balance Method (HBM) [9] [10] are preferred owing to their higher computational efficiency. Determining the local stability of a periodic solution is particularly interesting in an engineering context since only stable solutions are experimentally encountered [11]. Moreover, a change in the stability can lead to significant, qualitative, and possibly dramatic changes in the system response. Bifurcations that indicate regime changes are largely studied [12] [13]. Efforts have been previously made by researchers for accurate detection of bifurcation points which include fold bifurcations (limit points) and branch point bifurcations. Since the Jacobian 
matrix becomes singular at these points, most of the proposed methods use this characteristic as additional constraint in so-called augmented systems defining bifurcation points [14] [15] [16].

At the design stage, a particular attention must be paid to the influence of parameters. A parametric analysis is often performed in order to find the best parameters that meet operating requirements and lead to optimal run. For instance, choosing appropriate parameters can avoid dangerous resonance phenomena by moving the resonance frequencies out of the operating frequency range or by decreasing the resonance amplitudes to acceptable levels. In vibration analysis, the resonance levels and frequencies are of primary interest in forced response investigations. Many works aim at finding the peak amplitudes in nonlinear dynamical systems. Petrov $[17,18]$ applies the HBM to compute the worst vibration cases of bladed disks with friction contact interfaces. In [19], Liao combines the shooting method and Floquet theory along with a Global Search algorithm to determine the resonant peak of nonlinear systems.

When stability is the main design criteria, such a parametric analysis is commonly achieved by computing a stability chart which contains the various stability boundaries of the system with respect to a bifurcation parameter. A very simple way to do that consists in computing the response curve of the system for several values of the chosen bifurcation parameter, then detecting the bifurcation points on these curves and, finally, gathering these bifurcation points to form the stability boundaries. However, this method is very consuming since it requires the computation of a lot of information among which only the limit points are of interest.

This paper presents an approach for direct parametric analysis of limit points of nonlinear dynamical systems. Instead of computing responses curves for several values of a given system parameter, the direct tracking of limit points is performed. Similar approaches can be found in the literature for quasi-static problems [20]. They are used here in the context of nonlinear dynamical systems and adapted for use with the HBM. The whole numerical procedure is based on the HBM and can be decomposed in three distinct steps. Firstly, a response curve is calculated by HBM combined with a continuation technique (Section 2) until a limit point is found by Floquet theory (Section 3). Then this starting limit point is used to initialize the direct following of the branch of limit points when a system parameter is varied (Section 4). Several numerical examples are addressed in Section 5 to demonstrate the capabilities and the performance of the proposed method. Finally, conclusions are drawn in the last section.

\section{EQUILIBRIUM PATH}

The equation of motion in the time domain for the nonlinear forced response of a rotating structure takes the form

$$
\mathbf{M} \ddot{\mathbf{x}}(t)+\mathbf{C}(\omega) \dot{\mathbf{x}}(t)+\mathbf{K}(\omega) \mathbf{x}(t)+\mathbf{f}_{n l}(\mathbf{x}, \dot{\mathbf{x}})=\mathbf{p}(\omega, t)
$$

where $\mathbf{x}(t)$ is a vector of displacements for all $n$ degrees of freedom (DOFs), M stands for the generalized $n \times n$ mass matrix, $\mathbf{C}(\omega)$ includes the damping matrix and matrices related to rotation effects which depend on $\omega$ such as gyroscopic or Coriolis effects, etc, and $\mathbf{K}(\omega)$ comprises the stiffness matrix and centrifugal effects when it relates to a model with 3-D finite elements, $\mathbf{f}_{n l}$ stands for the nonlinear forces, $\mathbf{p}(\omega, t)$ is a vector of external excitation forces which, in our case, is periodic (unbalance force for rotating machines), and $\omega$ is the excitation frequency.

The HBM is utilized for its higher computational efficiency compared to classical time domain methods. It is based on the assumption that a periodic excitation leads to a periodic response. The displacements, nonlinear forces and external forces are therefore represented by Fourier series truncated at order $N$

$$
\begin{aligned}
\mathbf{x}(t) & =\mathbf{X}^{0}+\sum_{k=0}^{N} \mathbf{X}_{c}^{k} \cos (k \omega t)+\mathbf{X}_{s}^{k} \sin (k \omega t) \\
\mathbf{f}_{n l}(t) & =\mathbf{F}^{0}+\sum_{k=0}^{N} \mathbf{F}_{c}^{k} \cos (k \omega t)+\mathbf{F}_{s}^{k} \sin (k \omega t) \\
\mathbf{p}(t) & =\mathbf{P}^{0}+\sum_{k=0}^{N} \mathbf{P}_{c}^{k} \cos (k \omega t)+\mathbf{P}_{s}^{k} \sin (k \omega t)
\end{aligned}
$$

and Fourier coefficients are gathered into vectors of size $M=$ $n(2 N+1)$

$$
\begin{aligned}
\mathbf{X} & =\left[\mathbf{X}^{0}, \mathbf{X}_{c}^{1}, \mathbf{X}_{s}^{1}, \ldots, \mathbf{X}_{c}^{N}, \mathbf{X}_{s}^{N}\right]^{T} \\
\mathbf{F}_{n l} & =\left[\mathbf{F}^{0}, \mathbf{F}_{c}^{1}, \mathbf{F}_{s}^{1}, \ldots, \mathbf{F}_{c}^{N}, \mathbf{F}_{s}^{N}\right]^{T} \\
\mathbf{P} & =\left[\mathbf{P}^{0}, \mathbf{P}_{c}^{1}, \mathbf{P}_{s}^{1}, \ldots, \mathbf{P}_{c}^{N}, \mathbf{P}_{s}^{N}\right]^{T}
\end{aligned}
$$

By substituting Eqs. (2)-(4) into (1) then applying a Galerkin procedure, the nonlinear differential Equation (1) is transformed into a nonlinear algebraic equation system of size $M$ in which the time dependency has been removed

$$
\mathbf{R}(\mathbf{X}, \omega, \lambda)=\mathbf{Z}(\omega) \mathbf{X}+\mathbf{F}_{n l}\left(\mathbf{X}, \lambda_{F}\right)-\mathbf{P}\left(\lambda_{p}\right)=\mathbf{0}
$$

where

$$
\begin{gathered}
\mathbf{Z}=\operatorname{diag}\left(\mathbf{K}, \mathbf{Z}_{1}, . . \mathbf{Z}_{j}, . . \mathbf{Z}_{N}\right) \\
\mathbf{Z}_{k}=\left[\begin{array}{cc}
\mathbf{K}(\omega)-j^{2} \omega^{2} \mathbf{M} & \omega \mathbf{C}(\omega) \\
-\omega \mathbf{C}(\omega) & \mathbf{K}(\omega)-j^{2} \omega^{2} \mathbf{M}
\end{array}\right]
\end{gathered}
$$

$\lambda_{F}$ and $\lambda_{p}$ are parameters of nonlinear and excitation forces that can be varied later for parametric analysis. Equation (6) represents the equilibrium residual in the frequency domain. It is solved with a Newton-Raphson's method which consists in correcting iteratively an initial solution $\mathbf{X}^{0}$

$$
\mathbf{X}^{k+1}=\mathbf{X}^{k}+\delta \mathbf{X}
$$


where the correction $\delta \mathbf{X}$ is given by the linearized equation at iteration $k$

$$
\mathbf{J}^{k} \delta \mathbf{X}=-\mathbf{R}^{k}
$$

where

$$
\mathbf{J}(\mathbf{X}, \omega, \lambda)=\frac{\partial \mathbf{R}}{\partial \mathbf{X}}=\mathbf{Z}(\omega)+\frac{\partial \mathbf{F}_{n l}}{\partial \mathbf{X}}
$$

is the Jacobian matrix, and the superscript $k$ indicates evaluation at iteration $k$, i.e. $\mathbf{R}^{k}=\mathbf{R}\left(\mathbf{X}^{k}\right)$ is the equilibrium residual at iteration $k$.

The Fourier coefficients of the nonlinear forces $\mathbf{F}_{n l}$ and their derivative $\frac{\partial \mathbf{F}_{n l}}{\partial \mathbf{X}}$ involved in the Newton-Raphson iterations are obtained by Alternating Frequency-Time (AFT) method [21]. The AFT scheme uses fast direct and inverse Fourier transforms to compute the nonlinear forces in the time domain and then switch back to the frequency domain for the reason that nonlinear forces are usually much easier to evaluate in the time domain than in the frequency domain. Once the Fourier coefficients $\mathbf{X}$ are obtained, the time solution can be computed with Eq. (2) and provides one point of the frequency-response curve. This curve plots the maximum amplitude of the periodic displacement $\mathbf{x}(t)$ with respect to the frequency $\omega$. It is also referred to as the equilibrium path or solution branch in the following.

Nonlinear systems often have several possible responses for a given excitation frequency $\omega$. The pseudo-arc length continuation method [22] combined with the abovementioned algorithm permits following the solution branch beyond limit points in order to obtain both stable and unstable parts of the response curve. Firstly a prediction step is performed in the direction tangent to the solution curve. Then, corrections are applied iteratively in the orthogonal direction until the residual $\mathbf{R}^{k}$ becomes smaller than a userdefined accuracy. As a consequence, $\omega$ becomes a new unknown and the corrections $\delta \mathbf{X}$ and $\delta \omega$ are given by the $M+1$ linear system

$$
\left[\begin{array}{cc}
\mathbf{J}^{k} & \frac{\partial \mathbf{R}^{k}}{\partial \omega} \\
\Delta \mathbf{X}^{T} & \Delta \omega
\end{array}\right]\left\{\begin{array}{l}
\delta \mathbf{X} \\
\delta \omega
\end{array}\right\}=\left\{\begin{array}{c}
-\mathbf{R}^{k} \\
0
\end{array}\right\}
$$

where $[\Delta \mathbf{X} \Delta \omega]$ stands for the unitary tangent vector and the last equation is thus a scalar constraint enforcing the orthogonality of the corrections. The derivative of $\mathbf{R}$ with respect to $\omega$ is immediate from Eq. (6)

$$
\frac{\partial \mathbf{R}}{\partial \omega}=\frac{\partial \mathbf{Z}}{\partial \omega} \mathbf{X}
$$

A solution $(\mathbf{X}, \omega)$ is usually obtained in a few iterations. It then serves as a starting point for the next continuation step.

\section{STABILITY ANALYSIS AND LOCALIZATION OF LIMIT POINTS}

Floquet theory is the most widely used method for evaluating the stability of periodic solutions. It can be applied either in the frequency domain through Hill's method [10] or in the time domain through the computation of the socalled monodromy matrix [13]. The latter is used here and the monodromy matrix is computed with the Newmark time integration scheme, since it has been shown to be the best compromise between computational time and accuracy [23]. It consists in integrating over one period of motion $\tau=2 \pi / \omega$ the linear differential system

$$
\mathbf{M z}(t)+\left(\mathbf{C}+\frac{\partial \mathbf{f}_{n l}}{\partial \dot{\mathbf{x}}}\right) \dot{\mathbf{z}}(t)+\left(\mathbf{K}+\frac{\partial \mathbf{f}_{n l}}{\partial \mathbf{x}}\right) \mathbf{z}(t)=\mathbf{0}
$$

with initial conditions $\mathbf{z}(0)=\left[\mathbf{I}_{n} \mathbf{0}_{n}\right], \dot{\mathbf{z}}(0)=\left[\begin{array}{ll}\mathbf{0}_{n} & \mathbf{I}_{n}\end{array}\right]$ and $\ddot{\mathbf{z}}(0)$ obtained by replacing $\mathbf{z}(0)$ and $\dot{\mathbf{z}}(0)$ in Eq. (14). $\mathbf{I}_{n}$ and $\mathbf{0}_{n}$ stand here for the identity and the null matrices of size $n \times n$. The monodromy matrix $\mathbb{M}$ is then given by

$$
\mathbb{M}=\left[\begin{array}{c}
\mathbf{z}(\tau) \\
\dot{\mathbf{z}}(\tau)
\end{array}\right]
$$

Using 256 or more time steps $t_{i}$ over the integration period usually provides satisfactory accuracy of the results. Given a periodic solution $(\mathbf{X}, \omega)$ in the frequency domain, obtained as described in previous section, the displacements are evaluated at these time steps with Eq. (2) along with the derivatives of $\mathbf{f}_{n l}$ involved in (14).

The eigenvalues $\Lambda$ of the monodromy matrix, also known as the Floquet multipliers, provide information on the stability of the periodic solution. If all the multipliers are inside the unit circle in the complex plane $(\operatorname{Re}(\Lambda), \operatorname{Im}(\Lambda))$, the corresponding solution is asymptotically stable. When a multiplier crosses the unit circle, there is a local bifurcation on the equilibrium path with a loss of stability. Depending on the way the multiplier crosses the unit circle permits the characterization of the type of bifurcation [13]: when one multiplier goes out of the unit circle through the positive real axis, there is a limit point (LP), also called fold bifurcation, or a branch point (BP) bifurcation; if a multiplier crosses the circle through the negative real axis, there is a period-doubling bifurcation; when a pair of complex conjugate multipliers leave the circle, there is a Neimark-Sacker (NS), also called generalized Hopf bifurcation, which indicates the transition from periodic to quasi-periodic solutions.

If only limit points are of interest, an efficient alternative to the computation of the monodromy matrix consists in monitoring the component $\Delta \omega$ of the predictor tangent vector. More precisely, a limit point is indicated by a change of sign of $\Delta \omega$ between two consecutive points on the equilibrium path, and the point $\left(\mathbf{X}^{0}, \omega^{0}\right)$ with the smallest absolute value of $\Delta \omega$ is used as a starting point for the precise localization of the limit point.

To locate limit points precisely, a constraint equation 
characterizing the singularity of $\mathbf{J}$

$$
\mathbf{J} \phi=\mathbf{0}
$$

with $\phi$ the eigenvector associated to the null eigenvalue of $\mathbf{J}$, is added to the equilibrium equation (6) in order to restrict the equilibrium path to singular points only [14], leading to the so-called extended system at iteration $k$

$$
\left[\begin{array}{ccc}
\mathbf{J}^{k} & \mathbf{0} & \frac{\partial \mathbf{R}^{k}}{\partial \omega} \\
\frac{\partial(\mathbf{J} \phi)^{k}}{\partial \mathbf{X}} & \mathbf{J}^{k} & \frac{\partial(\mathbf{J} \boldsymbol{\phi})^{k}}{\partial \omega} \\
\mathbf{0}^{T} & 2 \phi^{k T} & 0
\end{array}\right]\left\{\begin{array}{c}
\delta \mathbf{X} \\
\delta \phi \\
\delta \omega
\end{array}\right\}=-\left\{\begin{array}{c}
\mathbf{R}^{k} \\
\mathbf{J}^{k} \phi^{k} \\
\left\|\phi^{k}\right\|^{2}-1
\end{array}\right\}
$$

The last normalization equation ensures a unique non trivial solution. A starting value for the eigenvector $\phi$ is obtained by performing an eigenvalue decomposition of the jacobian $J\left(\mathbf{X}^{0}, \omega^{0}\right)$ and selecting the eigenvector corresponding to the eigenvalue with the smallest absolute magnitude. The higher order derivatives involved in the linearization of Eq. (16) are conveniently computed in the frequency domain by means of finite differences [15]

$$
\begin{aligned}
& \frac{\partial(\mathbf{J} \phi)}{\partial \mathbf{X}} \simeq \frac{1}{\varepsilon}[\mathbf{J}(\mathbf{X}+\varepsilon \phi, \omega)-\mathbf{J}(\mathbf{X}, \omega)] \\
& \frac{\partial(\mathbf{J} \phi)}{\partial \omega} \simeq \frac{1}{\varepsilon}[\mathbf{J}(\mathbf{X}, \omega+\varepsilon)-\mathbf{J}(\mathbf{X}, \omega)] \phi
\end{aligned}
$$

Since $\phi$ is constant and does not depend on $\omega$ for a fixed configuration, and since only $\mathbf{Z}(\omega)$ depends on $\omega$ in Eq. (11), the derivative with respect to $\omega$ can be advantageously computed by

$$
\frac{\partial(\mathbf{J} \phi)}{\partial \omega}=\frac{\partial \mathbf{J}}{\partial \omega} \phi=\frac{\partial \mathbf{Z}}{\partial \omega} \phi
$$

The augmented system (17) is not singular, so it can be sent to a direct linear solver. However, when using many harmonics or for a large system with many degrees of freedom, this method becomes prohibitive. A block elimination algorithm [24] is then preferred. Doing so, J becomes the only matrix involved in the solving process. However, this matrix becomes singular when approaching to the limit points. It is thus very ill-conditioned during the last iterations of the Newton-Raphson procedure. To overcome this problem, a penalty term is added to matrix $\mathbf{J}$ in order to eliminate the singularity [25]

$$
\hat{\mathbf{J}}^{k}=\mathbf{J}^{k}+s \mathbf{e}_{i} \mathbf{e}_{i}^{T}
$$

and the first term $\mathbf{R}^{k}$ of the r.h.s. of (17) is replaced by $\mathbf{R}^{k}+s \mathbf{e}_{i}\left(\mathbf{e}_{i}^{T} \mathbf{X}\right)$, where $\mathbf{e}_{i}$ is a unit vector with $i$-th component equal to $1, i$ is the index of the largest real component of the eigenvector $\phi$ and $s$ is a constant chosen equal to the mean value of $\operatorname{diag}(\mathbf{J})$. The first equation of (17) is then decomposed in three linear systems with the same matrix $\hat{\mathbf{J}}$ and an extra variable $\beta_{1}$ is introduced

$$
\begin{aligned}
\hat{\mathbf{J}}^{k} \mathbf{a}_{1} & =-\mathbf{R}^{k} \\
\hat{\mathbf{J}}^{k} \mathbf{a}_{2} & =-\frac{\partial \mathbf{R}^{k}}{\partial \omega} \\
\hat{\mathbf{J}}^{k} \mathbf{a}_{3} & =s \mathbf{e}_{i} \\
\beta_{1} & =\mathbf{e}_{i}^{T} \delta \mathbf{X}
\end{aligned}
$$

$\delta \mathbf{X}$ is then given by

$$
\delta \mathbf{X}=\mathbf{a}_{1}+\mathbf{a}_{2} \delta \omega+\mathbf{a}_{3} \beta_{1}
$$

Replacing Eq. (26) in the second equation of (17) yields

$$
\begin{aligned}
\hat{\mathbf{J}}^{k} \mathbf{b}_{1} & =-\frac{\partial(\mathbf{J} \phi)^{k}}{\partial \mathbf{X}} a_{1}-\mathbf{J}^{k} \phi^{k} \\
\hat{\mathbf{J}}^{k} \mathbf{b}_{2} & =-\frac{\partial(\mathbf{J} \phi)^{k}}{\partial \mathbf{X}} a_{2}-\frac{\partial(\mathbf{J} \phi)^{k}}{\partial \omega} \\
\hat{\mathbf{J}}^{k} \mathbf{b}_{3} & =-\frac{\partial(\mathbf{J} \phi)^{k}}{\partial \mathbf{X}} a_{3}
\end{aligned}
$$

and $\delta \phi$ is given by

$$
\delta \phi=\mathbf{b}_{1}+\mathbf{b}_{2} \delta \omega+\mathbf{b}_{3} \beta_{1}+\mathbf{a}_{3} \beta_{2}
$$

where

$$
\beta_{2}=\mathbf{e}_{i}^{T} \delta \phi
$$

Finally, replacing Eqs. (26) and (30) in the third equation of (17) and in Eqs. (25) and (31) gives the $3 \times 3$ linear system

$$
\left[\begin{array}{ccc}
2 \phi^{k T} \mathbf{b}_{2} & 2 \phi^{k T} \mathbf{b}_{3} & 2 \phi^{k T} \mathbf{a}_{3} \\
\mathbf{e}_{i}^{T} \mathbf{a}_{2} & \mathbf{e}_{i}^{T} \mathbf{a}_{3}-1 & 0 \\
\mathbf{e}_{i}^{T} \mathbf{b}_{2} & \mathbf{e}_{i}^{T} \mathbf{b}_{3} & \mathbf{e}_{i}^{T} \mathbf{a}_{3}-1
\end{array}\right]\left\{\begin{array}{l}
\delta \omega \\
\beta_{1} \\
\beta_{2}
\end{array}\right\}=\left\{\begin{array}{c}
1-\left\|\phi^{k}\right\|^{2}-2 \phi^{k T} \mathbf{b}_{1} \\
-\mathbf{e}_{i}^{T} \mathbf{a}_{1} \\
-\mathbf{e}_{i}^{T} \mathbf{b}_{1}
\end{array}\right\}
$$

This system provides $\delta \omega, \beta_{1}, \beta_{2}$ from which $\delta \mathbf{X}$ and $\delta \phi$ can be computed with Eqs. (26) and (30).

The six linear systems (22)-(24) and (27)-(29) are solved with a factor-solve method using the LU-decomposition of $\hat{\mathbf{J}}$ followed by six forward/back substitutions of size $M$ instead of one direct solving of size $2 M+1$. This method makes it possible to exploit the sparsity of $\mathbf{J}$ originating from finite difference or finite element discretization, and reduces the effort of implementation by reusing standard Newton-Raphson routines corresponding to Eq. (10).

\section{PARAMETRIC CONTINUATION OF LIMIT POINTS}

The stability boundary of the system can be obtained by collecting the bifurcation points when a system parameter $\lambda$ (either $\lambda_{F}$, or $\lambda_{p}$ ) is varied. To this end, the simplest 
approach consists in computing the response curve sequentially for each value of the system parameter $\lambda$ and to detect the limit points with the algorithms presented in Sections 2 and 3. A much more efficient procedure consists in computing the response curve for a given value of $\lambda$, to detect a starting limit point on this curve, then to directly follow the branch of limit points when $\lambda$ is varied, without computing the other response curves. This procedure is described below in the case of limit points.

Once a starting limit point $\left(\mathbf{X}^{0}, \omega^{0}, \phi^{0}\right)$ is precisely detected along the response curve corresponding to $\lambda=\lambda^{0}$, its variation with respect to $\lambda$ is directly followed with a continuation method similar to that described in Section 2. In order to do this, $\lambda$ is considered as a new unknown and an arc-length equation is added to the augmented system (17). The predictor step calculates an approximated solution along the tangent unitary vector $[\Delta \mathbf{X} \Delta \omega \Delta \lambda]$, then the corrector step applies Newton-Raphson corrections in the orthogonal direction. This results in adding to system (17) one row corresponding to the orthogonality condition and one column corresponding to the new unknown $\lambda$. The following extended system for iteration $k$ of Newton-Raphson corrections is thus obtained

$$
\left[\begin{array}{cccc}
\mathbf{J}^{k} & \mathbf{0} & \frac{\partial \mathbf{R}^{k}}{\partial \omega} & \frac{\partial \mathbf{R}^{k}}{\partial \lambda} \\
\frac{\partial(\mathbf{J} \phi)^{k}}{\partial X} & \mathbf{J}^{k} & \frac{\partial(\mathbf{J} \phi)^{k}}{\partial \omega} & \frac{\partial(\mathbf{J} \phi)^{k}}{\partial \lambda} \\
\mathbf{0} & 2 \phi^{k T} & 0 & 0 \\
\Delta \mathbf{X}^{T} & \mathbf{0} & \Delta \omega & \Delta \lambda
\end{array}\right]\left\{\begin{array}{c}
\delta \mathbf{X} \\
\delta \phi \\
\delta \omega \\
\delta \lambda
\end{array}\right\}=-\left\{\begin{array}{c}
\mathbf{R}^{k} \\
\mathbf{J}^{k} \phi^{k} \\
\left\|\phi^{k}\right\|^{2}-1 \\
0
\end{array}\right\}
$$

where the new derivatives can be calculated analytically

$$
\frac{\partial \mathbf{R}}{\partial \lambda}=\frac{\partial \mathbf{F}_{N L}}{\partial \lambda}-\frac{\partial \mathbf{P}}{\partial \lambda} \quad \frac{\partial(\mathbf{J} \phi)}{\partial \lambda}=\frac{\partial^{2} \mathbf{F}_{N L}}{\partial \mathbf{X} \partial \lambda} \phi
$$

or by finite differences as in (19), and $\left(\mathbf{X}^{0}, \omega^{0}, \phi^{0}, \lambda^{0}\right)$ are used as initial conditions. The block elimination algorithm detailed in Section 3 can be used to reduce the computational cost. This time, it requires the LU-decomposition of $\hat{\mathbf{J}}$ followed by eight forward/back substitutions of size $M$ instead of one direct solving of the $2 M+2$ system (33). In terms of computational cost, a step of LP continuation is roughly equivalent to the precise detection of a LP (Section 3 ) and, for large systems, it is barely more costly than the simple continuation of the response curve (Section 2) since in this case almost all the CPU time is devoted to the decomposition of $\hat{\mathbf{J}}$.

\section{NUMERICAL RESULTS}

Numerical simulations are presented in this section in order to demonstrate the validity and the effectiveness of the proposed approach.

\subsection{Duffing oscillator}

The Duffing oscillator is a single-degree of freedom system with a nonlinear restoring force proportional to the cube

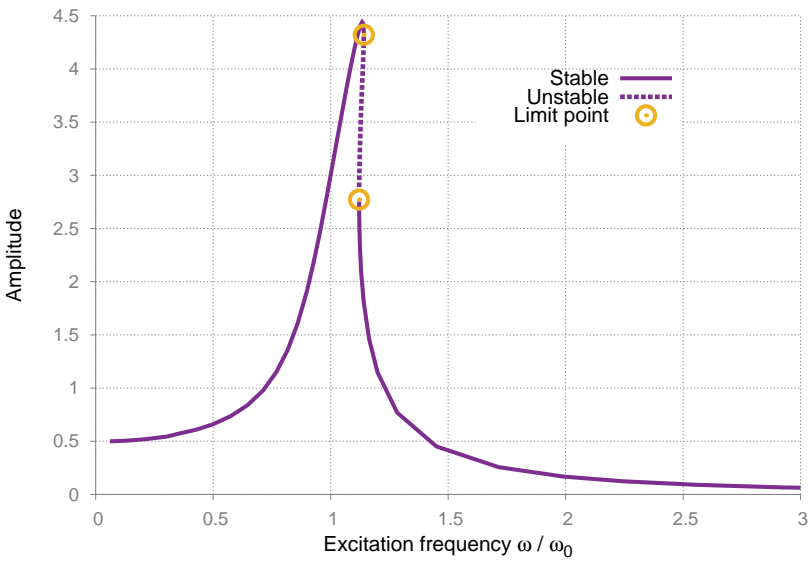

Fig. 1. Frequency response of the Duffing system for $\alpha=0.02$.

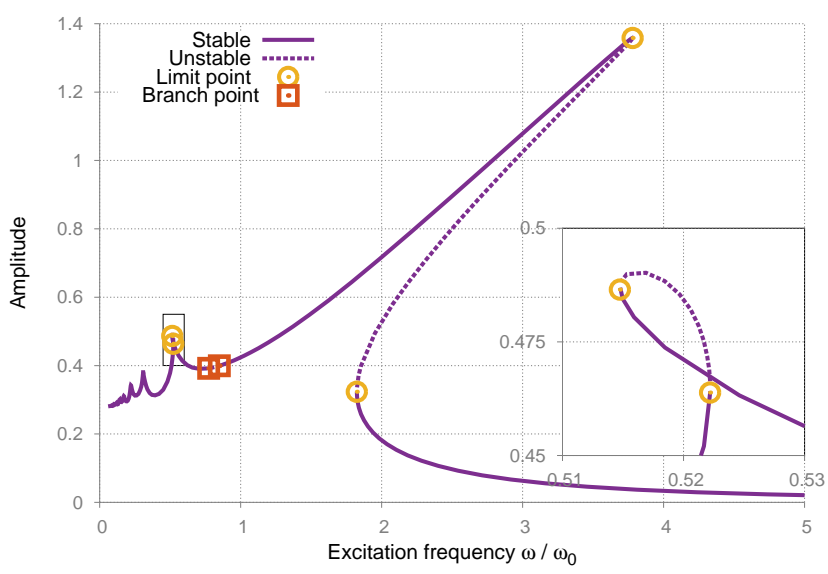

Fig. 2. Frequency response of the Duffing system for $\alpha=10$.

of the displacement. The system is described by the differential equation

$$
\ddot{x}(t)+2 \zeta \dot{x}(t)+\omega_{0}^{2} x(t)+\alpha x(t)^{3}=p_{0} \cos (\omega t)
$$

where $\alpha$ is the nonlinearity coefficient, and $p_{0}$ is the excitation amplitude.

Using the HBM continuation with the excitation frequency as the continuation parameter, the frequency response computed with $2 \zeta=0.1, \omega_{0}=1, p_{0}=0.5, \alpha=0.02$ and $N=3$ harmonics is shown in Fig.1. A slightly hardening resonance curve can be observed, where the solid and dotted lines stand for stable and unstable solutions respectively. The two limit points (indicated by circles) are obtained by monitoring a change of sign of the component $\Delta \omega$ of the predictor tangent vector and double-checked by examining the evolution of the Floquet multipliers. In addition, the stable parts of this response curve are in excellent agreement with the results obtained by direct time integration which are not presented here for the sake of conciseness.

For stronger nonlinear effect $(\alpha=10)$, super-harmonic resonances are clearly visible at lower frequency range, and additional limit points accompanied by stability changes are found as shown in the zoom frame in Fig.2. It should be 


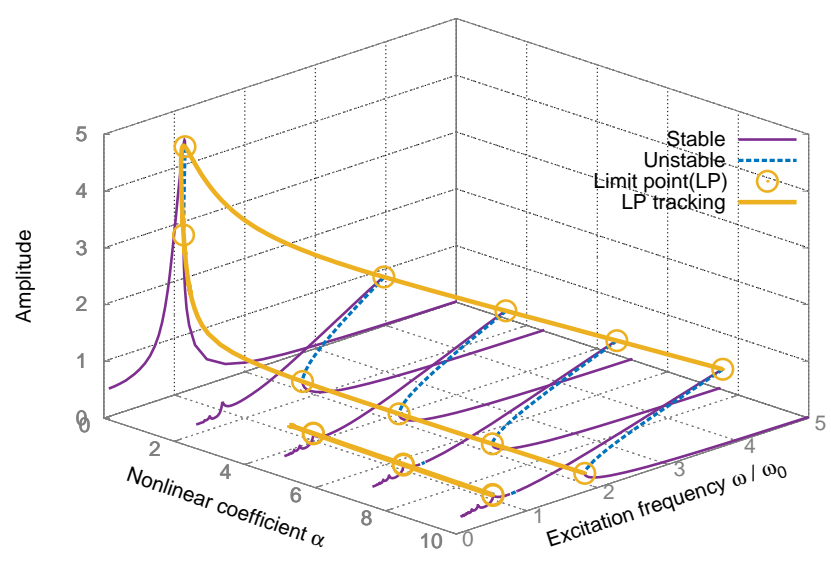

Fig. 3. Limit points tracking for $\alpha \in\left[\begin{array}{ll}0 & 10\end{array}\right]$.

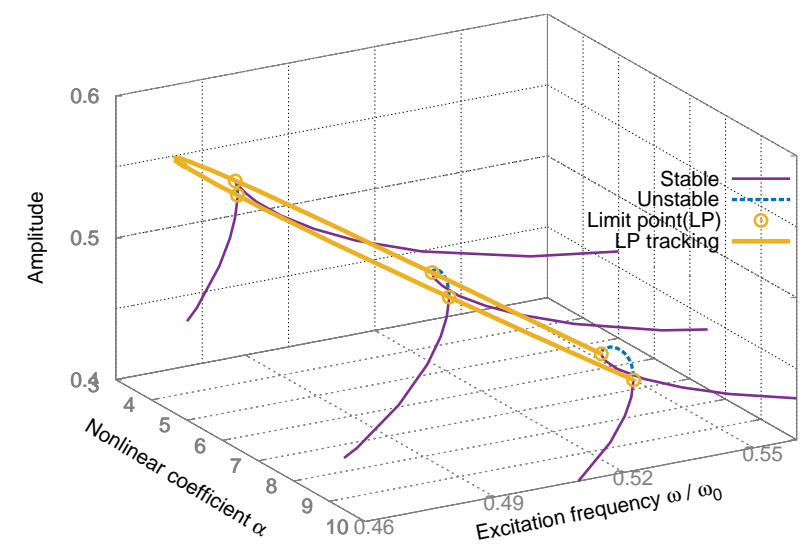

Fig. 4. Zoom of limit points tracking

noted that a higher number of harmonics $N=19$ is necessary to obtain the super-harmonic resonances. Two BP bifurcations where also detected by monitoring the Floquet multipliers. They indicate bifurcation branches emerging from the fundamental response curve.

As explained in Section 4, the limit points can be directly followed when the nonlinear coefficient $\alpha$ is varied. To this end, a response curve was first followed for a fixed value $\alpha=2$ until a limit point was detected. Then, this limit point was used as a starting point for the limit point tracking. The resulting branch of limit points is plotted in Fig.3. The response curves plotted in Fig. 3 for some values of $\alpha$ were not used for the computations. They are presented here to make the interpretation of the 3D-plot easier and to show that the direct LP tracking works as expected and is accurate. The second branch of limit points, magnified in Fig.4, corresponds to the limit points of the super-harmonic loops. It was obtained by using a starting limit point on the response curve for $\alpha=10$.

The projection of these branches of limit point on the $\alpha-\omega$ plane is plotted in Fig.5. It corresponds to a direct parametric analysis obtained without computing all the response curves of the system. This parametric analysis can be used to enhance the design and performances of the system

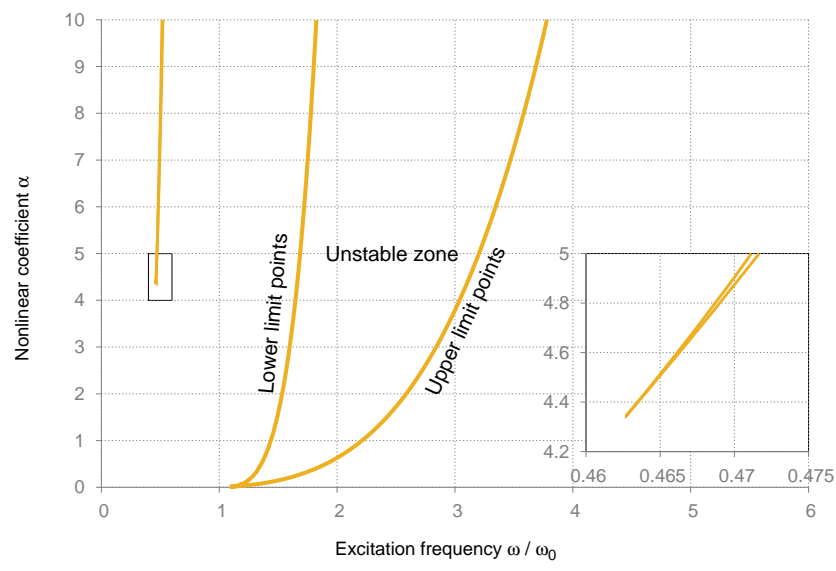

Fig. 5. Projections of limit points tracking

and, more generally, provides useful information about the dynamic behaviour of the system. From this projection, it can be observed that the lower and upper limit points merge for $\alpha \simeq 0.0106$. Under this value, the behaviour of the system is linear with only one stable solution for each frequency. Above this value, the response curves are of hardening type and the lower and upper LP branches define the boundary of the so-called unstable zone in which the solution is bistable. For instance, the intersection of the horizontal line $\alpha=10$ with the lower and upper LP branches indicates two limit points for $\omega / \omega_{0}=1.82$ and 3.78. Amplitude jumps can thus be expected at these frequencies during experimental frequency sweeps. The second branch of limit points emerges near $\alpha \simeq 4.345$. As a consequence, super-harmonic loops will exist only for $\alpha$ above this value. Tracking the upper limit points also gives information on the frequency shift of the resonance peak induced by the level of nonlinearity. Similarly, using the projection on the $\alpha$-Amplitude plane provides information on the reduction of vibration amplitude induced by the level of nonlinearity.

\subsection{Nonlinear Jeffcott rotor}

The second test case is a modified Jeffcott rotor which can interact with a stator modelled by a stiffness [26] [27]. The rotor is made of a weightless shaft carrying a disk with mass $m$ at the middle of the span. The clearance between the rotor and the stator is denoted by $h$. The stator, which is rigidly fixed, has an elastic contact surface modelled as a symmetrical set of radial springs with isotropic stiffness $k_{c}$. The fundamental motion of the disk is governed by the following equations

$$
\begin{aligned}
& m \ddot{x}+c \dot{x}+k x+k_{c}\left(1-\frac{h}{r}\right)\left(x-\mu y \operatorname{sign}\left(v_{r e l}\right)\right)=p_{b} \omega^{2} \cos \omega t \\
& m \ddot{y}+c \dot{y}+k y+k_{c}\left(1-\frac{h}{r}\right)\left(\mu x \operatorname{sign}\left(v_{r e l}\right)+y\right)=p_{b} \omega^{2} \sin \omega t
\end{aligned}
$$

where $k$ is the stiffness of the shaft, $r=\sqrt{x^{2}+y^{2}}$ is the radial displacement of the disk, $p_{b}$ is the mass unbalance amplitude and $v_{r e l}=\left(\frac{x}{r} \dot{y}-\frac{y}{r} \dot{x}\right)+R_{d i s c} \omega$ is the relative velocity between 


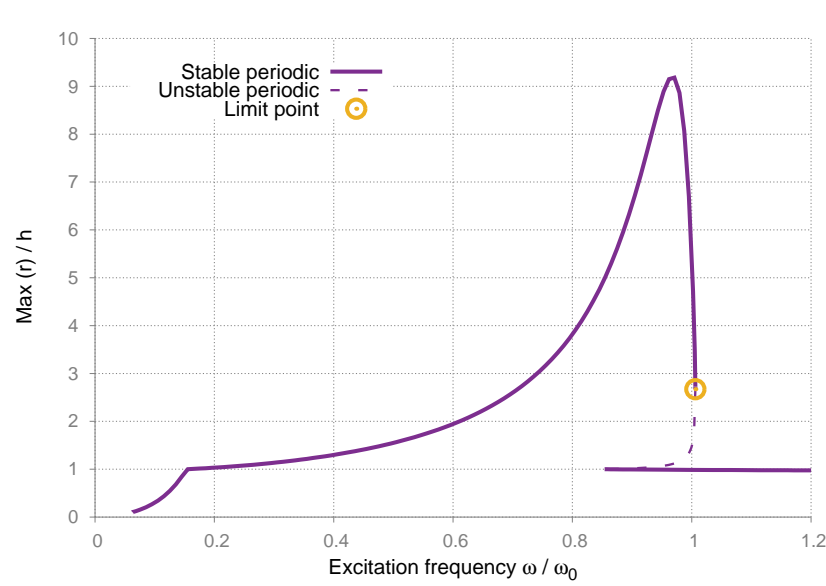

Fig. 6. Forced response of the Jeffcott rotor for $\mu=0$.

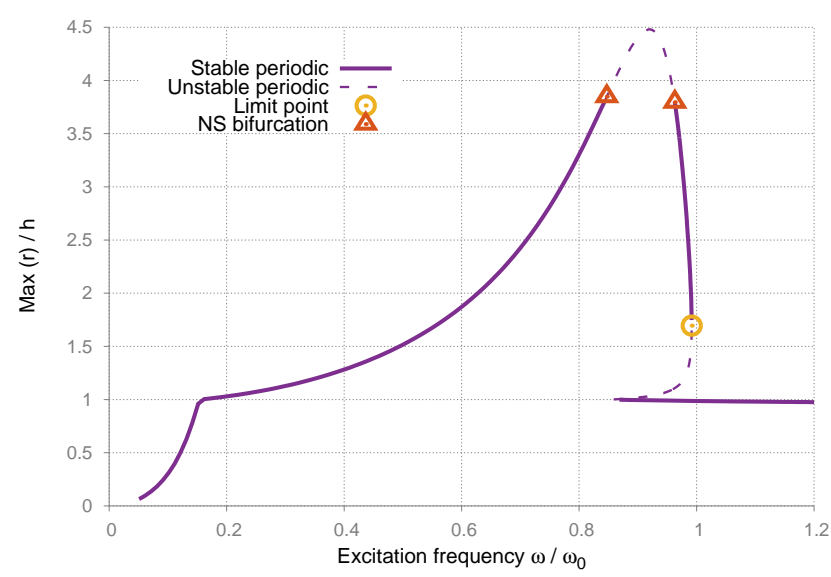

Fig. 7. Forced response of the Jeffcott rotor for $\mu=0.11$.

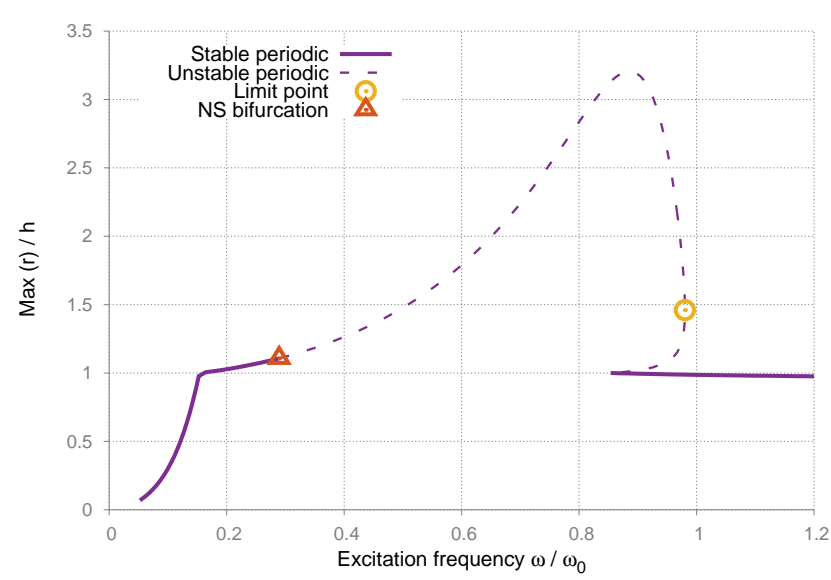

Fig. 8. Forced response of the Jeffcott rotor for $\mu=0.2$.

the rotor and the stator at the contact point. When $r<h$, there is no rub between the rotor and the stator, $k_{c}=0$.

All the calculations were carried out with the same set of parameters $m=1, c=5, k=100, k_{c}=2500, h=0.105, p_{b}=$ $0.1, R_{\text {disc }}=20 h, \omega_{0}=\sqrt{k_{c} / m}=50$, except that the friction coefficient $\mu$ was considered as the varying parameter. Based on comparison with results obtained by time integration, $N=$

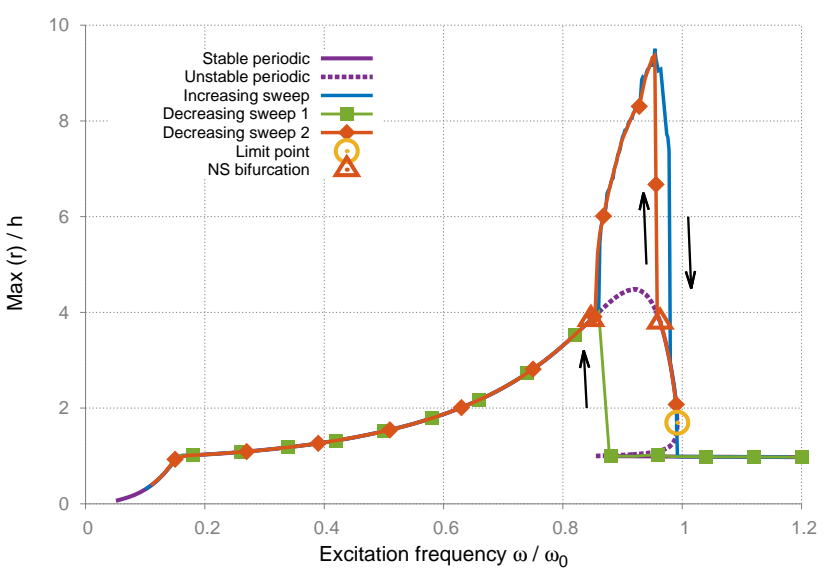

Fig. 9. Forced response of the Jeffcott rotor for $\mu=0.11$, comparison with time integration for increasing and decreasing frequency sweeps.

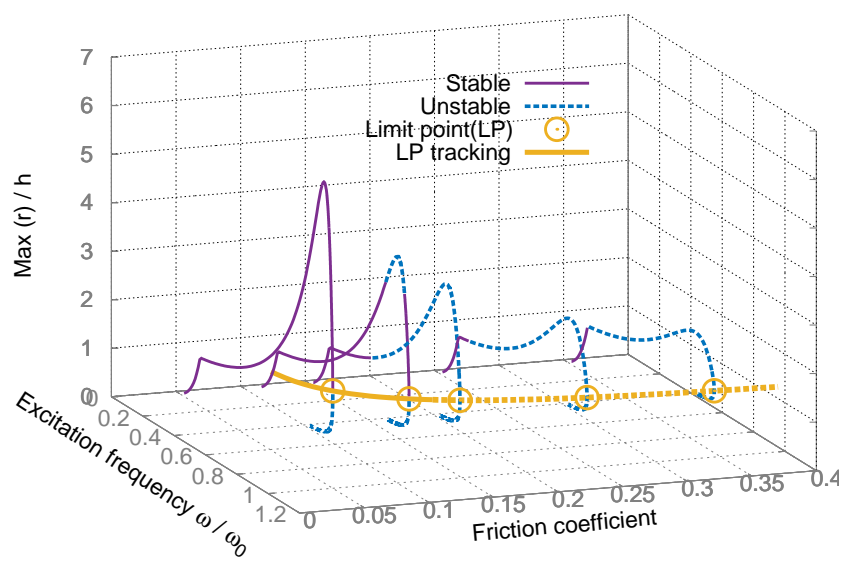

Fig. 10. Limit points tracking of the Jeffcott rotor with varying friction coefficient $\mu$.

15 harmonics were used to ensure a good accuracy of the HBM computations.

Firstly, the dimensionless response curve of the system as a function of $\omega / \omega_{0}$ is plotted in Fig. 6 for $\mu=0$. Both the change of sign of the component $\Delta \omega$ of the predictor tangent vector and the Floquet multipliers were monitored for the stability analysis. Solid and dotted lines indicate the stable and unstable branches respectively, while circles are used for limit points. The rotor-stator contact occurs for $r / h=1$, then synchronous full annular rub motion takes place for higher values of $\omega / \omega_{0}$. For this case, one limit point was found on the full annular rub part of the response curve. The motion is stable before contact and remains stable during the full annular rub until the limit point is reached for $\omega / \omega_{0} \simeq 1$. At this point, an amplitude jump is expected in the experimental response.

Other calculations were carried out for $\mu=0.11$ and $\mu=0.2$. As observed in Fig.7 for $\mu=0.11$, the response does not remain stable until the limit point is reached. Indeed, stability is lost between $\omega / \omega_{0}=0.82$ and $\omega / \omega_{0}=0.95$. This loss of stability is not related to limit point but to Neimark- 
Sacker bifurcations. Indeed, a pair of complex-conjugate Floquet multipliers was found to leave then re-enter the unit circle at these points. For $\mu=0.2$ (Fig.8), the response becomes unstable at $\omega / \omega_{0}=0.28$, where a NS bifurcation occurs, and remains unstable as long as the rotor and the stator remain in contact. Even though a limit point is found, it does not indicate a stability change in this case because there is still a pair of complex-conjugate Floquet multipliers, corresponding to the NS bifurcation, outside the unit circle.

In order to validate the calculations by HBM and the stability analysis, time integrations with increasing and decreasing frequency sweeps were carried out. In Fig.9, the blue curve corresponds to an increasing frequency sweep for $\omega / \omega_{0} \in[0.1-1.2]$. For the decreasing frequency sweep, two different sets of initial conditions were chosen in order to obtain all the stable parts of the response curve. The first decreasing frequency sweep (green curve) was initiated at $\omega / \omega_{0}=1.2$ while the second one (orange curve) was initiated at $\omega / \omega_{0}=0.99$ with an initial position of the rotor $\left(x_{0}, y_{0}\right)=(0.5 h, 0)$. Periodic stable responses predicted by HBM and time integration are identical, and amplitude jumps are observed in the time response when a stability loss is predicted by HBM, either at NS bifurcations or limit points. As predicted by the Floquet theory, a branch of quasiperiodic motion is observed between the two NS bifurcations. This stable branch corresponds to partial rub motion. In this range of frequency, quasi-periodic partial rub motion or motion without contact can take place depending on the initial conditions.

Similar to the Duffing oscillator example, the continuation procedure detailed in Section 4 was used to directly follow the branch of limit points. Here, the friction coefficient $\mu$ was used as additional variable parameter and the continuation was started from the limit point obtained for $\mu=0$. The resulting branch of limit points is plotted in Fig.10. Response curves for several values of $\mu$ are also represented in this 3D-plot to facilitate interpretation of the results. For an $10^{-6}$ accuracy, 32 adaptive continuation steps with an average number of 4 iterations per step were necessary to compute the response curve for $\mu=0$ and reach the limit point. Then, 65 adaptive steps were performed for the direct LP tracking. For this example, the CPU time for a LP tracking step appears to be 1.6 times higher than for a simple step on the response curve. Thus, the CPU time for the complete LP tracking is equivalent to the CPU time for 3 response curves and LP detections.

A stability analysis was also conducted during the limit point continuation in order to determine whether they correspond to a change of stability in the response curve as in Fig.6 or not as in Fig.8. These two cases are represented by solid and dotted lines respectively. As observed on the LP tracking, the solid line changes into a dotted line for $\mu \simeq 0.1285$. Under this value, the response curve is stable before reaching the limit point, potentially with a quasiperiodic part delimited by two NS bifurcations. Above this value, the periodic response curve is totally unstable between the remaining NS bifurcation and the limit point and there is a range of frequency in which the quasi-periodic partial rub

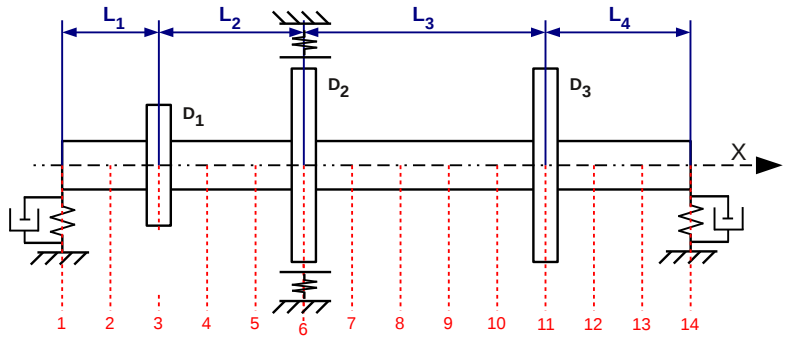

Fig. 11. Multi-DOFs FE rotor [23]

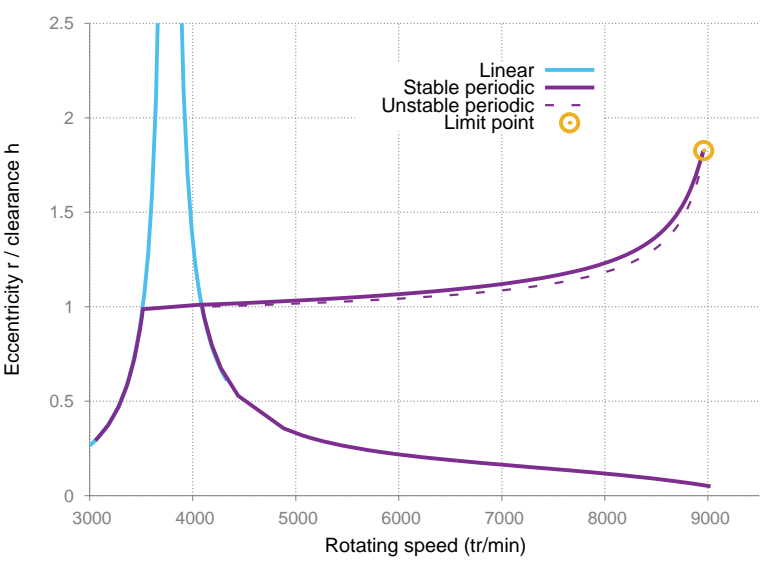

Fig. 12. FE rotor : Forced response at node 6 for $\mu=0$

is the only stable motion.

\subsection{Finite element nonlinear rotor}

A finite element (FE) nonlinear rotor model [28] was also tested (see Fig.11). This rotor is composed of 13 twonode beam elements, 3 rigid one-node disks and 2 one-node bearings. All shaft elements have the same length, with $L_{1}=0.2 \mathrm{~m}, L_{2}=0.3 \mathrm{~m}, L_{3}=0.5 \mathrm{~m}, L_{4}=0.3 \mathrm{~m}$ and the shaft diameter is $0.1 \mathrm{~m}$. Each node contains the four classical bending DOFs : two lateral displacements $u_{x}$ and $u_{y}$ and two rotations $\theta_{x}$ and $\theta_{y}$. The total number of DOFS is $n=56$. The two linear isotropic bearings, with stiffness and damping characteristics $k_{x x}=k_{y y}=6.10^{7} \mathrm{~N} . \mathrm{m}^{-1}, c_{x x}=c_{y y}=600 \mathrm{~N} . \mathrm{s} . \mathrm{m}^{-1}$, are located at both ends of the rotor. The disks are located at nodes 3, 6 and 11. Each disk is modelled with two identical mass and two identical mass moments of inertia for translational and rotational DOFs respectively. The disks and shaft are made of steel. The aim is to calculate the response to a mass unbalance excitation of magnitude $0.02 \mathrm{~kg} . \mathrm{m}$ located on disk D2. Contact is possible between disk D2 and a circular, static, rigidly fixed stator. The stator elasticity is modelled by springs with isotropic stiffness $k_{c}$ ten times higher than the bearing stiffness. The contact model is identical to that described in section 5.2 with an initial clearance $h=1 \mathrm{~mm}$. The equation of motion takes a form similar to Eq.(1) where $f_{n l}$ represents the contact forces between the second disk and the stator.

The unbalance mass response is calculated using the FE solver Cast3M [29]. The freely available version of Cast $3 \mathrm{M}$ can compute the mass unbalance response of a rotor by means of transient simulations. Based on these exist- 
ing capabilities, the HBM and arc-length continuation, the computation of the monodromy matrix, as well as the extended system and associated block elimination algorithm for the LP tracking presented in the previous sections, have been implemented in Cast3M using Gibiane command language. Based on comparison with time integration, it is found that $N=7$ harmonics are sufficient to ensure the convergence of the HBM algorithm. The size of the nonlinear algebraic HBM system (6) is thus $M=n(2 H+1)=840$.

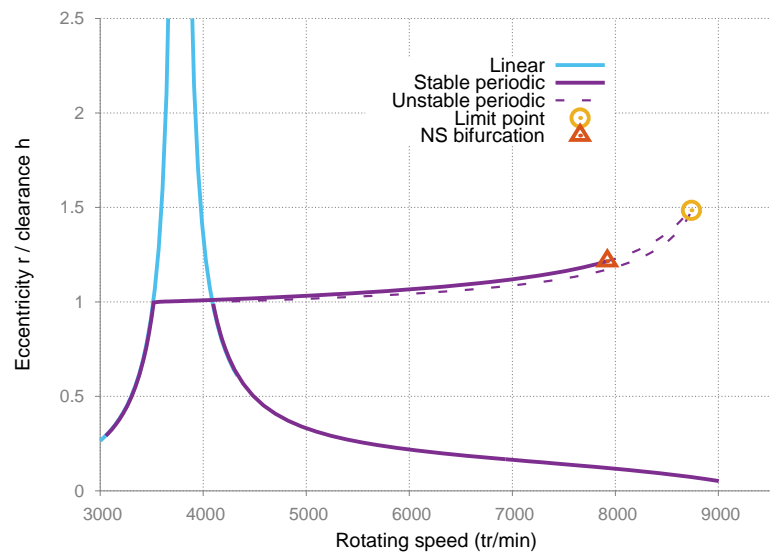

Fig. 13. FE rotor : Forced response at node 6 for $\mu=0.03$

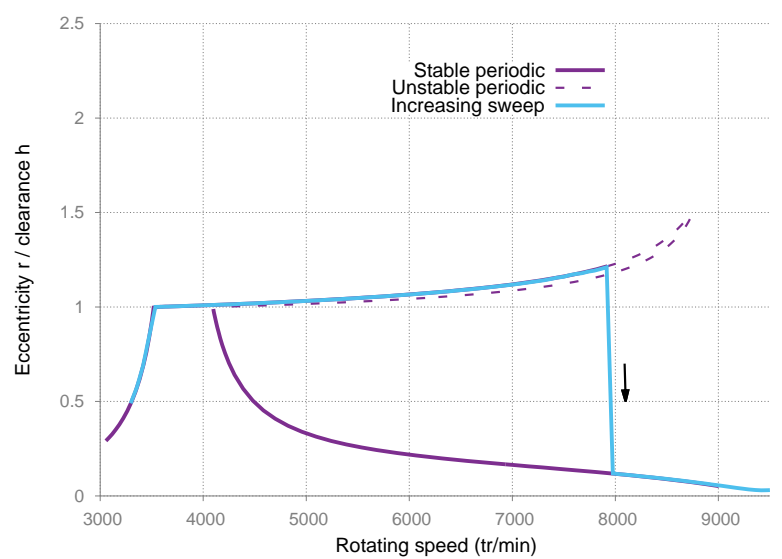

Fig. 14. FE rotor: Forced response at node 6 for $\mu=0.03$, comparison with time integration for increasing sweep.

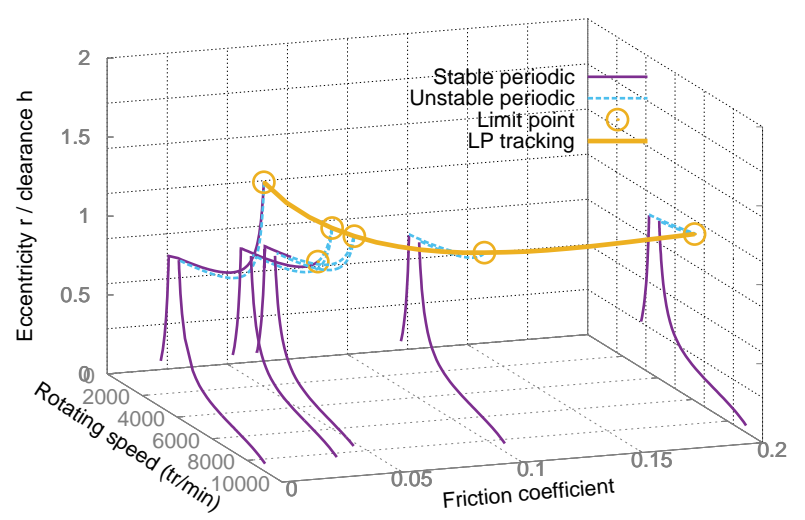

Fig. 15. FE rotor : Limit points tracking with varying friction coefficient $\mu$.
The size of the extended system (33) for LP tracking is then $2 M+2=1682$. However, the computational cost remains limited through the use of a block elimination algorithm as explained in Section 3.

The non-dimensional eccentricity $r / h$ and the rotating speed $\omega$ are used for the response curves. For $\mu=0$, the stable and unstable branches of the response are plotted in Fig.12. The blue line stands for linear response. Since the nonlinear forces are nil without contact, the linear and nonlinear responses coincide when $r<h$. The rotor-stator contact occurs when $R / h=1$, then synchronous full annular rub motion takes place. For this case, one limit point was found. The motion is stable before contact and remains stable during the full annular rub until the limit point is reached for $\omega \simeq 9000 \mathrm{tr} / \mathrm{min}$. At this point, an amplitude jump is expected in the experimental response, which can have a damaging effect on the rotor. $\mathrm{c}$ The response for $\mu=0.03$ is plotted in Fig.13. As for the Jeffcott rotor, a NS bifurcation is detected before the limit point. This bifurcation indicates the transition from the full annular rub motion to a quasi-periodic partial rub motion (not represented here). Between the NS bifurcation an the limit point, the only stable motions are the quasi-periodic partial rub and the motion without contact on the lower part of the response curve. This is confirmed by a comparison with time integration presented in Fig.14. As observed, an amplitude jump occurs during the increasing sweep when the periodic solution calculated by HBM is no longer stable.

The LP tracking with friction coefficient $\mu$ used as additional variable parameter is plotted in Fig. 15 for $\mu \in[0-0.2]$. The LP continuation was initiated with the limit point obtained for $\mu=0$. For this example, the CPU time for a LP tracking step appears to be only 1.8 times higher than for a simple step on the response curve.

\section{CONCLUSIONS}

An efficient frequency-domain method for fast parametric stability analysis of nonlinear dynamic systems has been presented. For this purpose, the direct tracking of limit points is performed. This limit point tracking is based on the combination of so-called extended systems and continuation technique in the framework of the Harmonic Balance Method (HBM). With only one computation, a complete branch of limit points is obtained, which provides the stability boundary with respect to systems parameters such as nonlinearity or excitation level. Numerical examples of increasing complexity have illustrated the capabilities and the performance of the proposed methodology.

For the examples considered here and, more generally, in most nonlinear systems, the characteristics of the response can be very complex due to other types of bifurcation (branch point, Neimark-Sacker). Thus, tracking limit points only is insufficient for a complete parametric analysis of the response. It can be supplemented by the tracking of NeimarkSacker bifurcations which gives the onset boundary of quasiperiodic motion. The extension of the proposed method to the tracking of such bifurcations is under progress. 


\section{ACKNOWLEDGMENTS}

The authors would like to acknowledge the support of CEA Saclay.

\section{References}

[1] Jacquet-Richardet, G., Torkhani, M., Cartraud, P., Thouverez, F., Baranger, T. N., Herran, M., Gibert, C., Baguet, S., Almeida, P., and Peletan, L., 2013. "Rotor to stator contacts in turbomachines. review and application”. Mech. Syst. Sig. Process., 40(2), pp. 401-420.

[2] Duran, C., Manin, L., Andrianoely, M.-A., Bordegaray, C., Battle, F., and Dufour, R., 2015. "Effect of rotorstator contact on the mass unbalance response". In Proc. IFToMM Int. Conf. on Rotordynamics, P. Pennacchi, ed., Vol. 21 of Mechanisms and Machine Science. Springer International Publishing, pp. 1965-1975.

[3] Chen, C., Dai, L., and Fu, Y., 2007. "Nonlinear response and dynamic stability of a cracked rotor". Commun. Nonlinear Sci. Numer. Simul., 12(6), pp. 1023 1037.

[4] Cheng, L., Li, N., Chen, X., and He, Z., 2011. “The influence of crack breathing and imbalance orientation angle on the characteristics of the critical speed of a cracked rotor". J. Sound Vib., 330(9), pp. 2031 - 2048.

[5] Khonsari, M. M., and Chang, Y. J., 1993. "Stability boundary of non-linear orbits within clearance circle of journal bearings". ASME J. Vib. Acoust., 115, pp. 303307.

[6] Brown, R. D., Addison, P., and Chan, A., 1994. "Chaos in the unbalance response of journal bearings". Nonlinear Dyn., 5(4), pp. 421-432.

[7] Dakel, M.-Z., Baguet, S., and Dufour, R., 2014. "Nonlinear dynamics of a support-excited flexible rotor with hydrodynamic journal bearings". J. Sound Vib., 333(10), pp. 2774-2799.

[8] Sundararajan, P., and Noah, S., 1997. "Dynamics of forced nonlinear systems using shooting/arc-length continuation method - application to rotor systems". ASME J. Vib. Acoust., 119, pp. 9-19.

[9] Kim, Y.-B., and Noah, S., 1996. "Quasi-periodic response and stability analysis for a non-linear jeffcott rotor". J. Sound Vib., 190(2), pp. 239 - 253.

[10] Von Groll, G., and Ewins, D. J., 2001. "The harmonic balance method with arc-length continuation in rotor/stator contact problems". J. Sound Vib., 241(2), pp. $223-233$.

[11] Nayfeh, A. H., and Mook, D. T., 1995. Nonlinear oscillations. Wiley New York.

[12] Berlioz, A., Dufour, R., and Sinha, S., 2000. "Bifurcation in a nonlinear autoparametric system using experimental and numerical investigations". Nonlinear Dyn., 23(2), pp. 175-187.

[13] Seydel, R., 2009. Practical Bifurcation and Stability Analysis. Interdisciplinary Applied Mathematics. Springer.

[14] Moore, G., and Spence, A., 1980. “The calculation of turning points of nonlinear equations". SIAM J. Numer. Anal., 17(4), pp. 567-576.

[15] Wriggers, P., and Simo, J. C., 1990. “A general procedure for the direct computation of turning and bifurcation points". Int. J. Numer. Meth. Engng., 30.

[16] Battini, J.-M., Pacoste, C., and Eriksson, A., 2003. "Improved minimal augmentation procedure for the direct computation of critical points". Comput. Methods Appl. Mech. Eng., 192(16-18), pp. 2169-2185.

[17] Petrov, E. P., 2007. "Direct parametric analysis of resonance regimes for nonlinear vibrations of bladed disks". J. TurboMach., 129(3), pp. 495-502.

[18] Petrov, E. P., 2009. "Method for sensitivity analysis of resonance forced response of bladed disks with nonlinear contact interfaces". J. Eng. Gas Turbines Power, $131(2)$.

[19] Liao, H., and Wang, J., 2013. "Maximization of the vibration amplitude and bifurcation analysis of nonlinear systems using the constrained optimization shooting method". J. Sound Vib., 332(16), pp. 3781-3793.

[20] Baguet, S., and Cochelin, B., 2002. "Stability of thinshell structures and imperfection sensitivity analysis with the Asymptotic Numerical Method". Rev. Européenne Elém. Finis, 11/2-3-4, pp. 493-509.

[21] Cameron, T. M., and Griffin, J. H., 1989. “An alternating frequency/time domain method for calculating the steady-state response of nonlinear dynamic systems". J. Appl. Mech., 56, pp. 149-154.

[22] Crisfield, M., 1981. "A fast incremental/iterative solution procedure that handles snap-through". Comput. Struct., 13(1), pp. 55-62.

[23] Peletan, L., Baguet, S., Torkhani, M., and JacquetRichardet, G., 2013. "A comparison of stability computational methods for periodic solution of nonlinear problems with application to rotordynamics". Nonlinear Dyn., 72(3), pp. 671-682.

[24] Keller, H. B., 1977. "Numerical solution of bifurcation and nonlinear eigenvalue problems". In Applications of Bifurcation Theory, P. H. Rabinowitz, ed., Academic Press, New York, pp. 359-384.

[25] Felippa, C. A., 1987. "Traversing critical points by penalty springs". Proc. NUMETA'87 Conf., Swansea, Wales, Nijhoff Pubs, Dordrecht, Holland.

[26] Jiang, J., 2009. "Determination of the global responses characteristics of a piecewise smooth dynamical system with contact". Nonlinear Dyn., 57(3), pp. 351-361.

[27] Peletan, L., Baguet, S., Torkhani, M., and JacquetRichardet, G., 2014. "Quasi-periodic harmonic balance method for rubbing self-induced vibrations in rotorstator dynamics". Nonlinear Dyn., 78(4), pp. 25012515.

[28] Lalanne, M., and Ferraris, G., 1998. Rotordynamics prediction in engineering. No. vol. 2 in Rotordynamics prediction in engineering. John Wiley.

[29] CAST3M, 2015. "Finite element software". CEA (French Atomic Energy Commission). http://wwwcast3m.cea.fr/. 